\title{
La teoría de los lugares centrales y su influencia en el desarrollo de las ciudades y en la economía regional
}

Mg. Econ. Wilson William TORRES DÍAZ ${ }^{37}$

\section{RESUMEN:}

Este artículo es importante porque deja un precedente sobre la necesidad de planificar el crecimiento y desarrollo de las ciudades en base a la identificación y satisfacción de necesidades, este genera la creación de mercados y permite una mejor calidad de vida para su población sino también la concentración de riqueza que haría sostenible su continuo crecimiento en el tiempo.

PALABRAS CLAVE: Lugar Central, Isotrópico, Centros De Servicio, Umbral De Demanda, Alcance Físico.

\section{ABSTRACT:}

This article is important because it sets a precedent about the need to plan for growth and development of cities based on identifying and satisfy the needs, it generated the creation of markets and allows better quality of life for their people but also to the concentration of wealth that would make their continued sustainable growth over time.

KEYWORDS: Central Place, Isotropic, Service Centers, Demand Threshold, Physical Reach.

REFLEXIÓN: Julia Hernández Aragón comenta que: “en el ámbito de la economía urbana, un elemento clave es la concepción de la ciudad, que deriva del proceso de urbanización. Este proceso significa el paso de la sociedad rural a una sociedad urbana. Se refiere al crecimiento acelerado de la población urbana en comparación con la población rural.

¿Por qué es importante desarrollar una ciudad? Porque toda ciudad puede convertirse en un foco económico de inversiones que beneficia a su población ¿Que se tiene que hacer para desarrollar una ciudad? ¿Qué se debe implementar

37 Cualquier consulta por favor escribir: Wilson.TorresD@urp.pe 
en esa ciudad? se tiene y se debe implementar una adecuada infraestructura según los mercados que existan ¿Cómo lograr el desarrollo de la población de una ciudad?, ¿Qué atrae una mayor cantidad de inversionistas y empresarios a una ciudad? los empresarios se ven a traídos por la rentabilidad y por la infraestructura que posea una ciudad para que atraiga a la inversión. Estas son preguntas para la planificación estratégica nacional dentro de una política económica que busca el crecimiento económico de la población de un país.

"La teoría de los Lugares Centrales de Walter Christaller" explica porque se distribuye y jerarquiza los espacios dentro de una ciudad. Aquí se discute el término "espacio isotrópico"; Un espacio isotrópico es un lugar donde las condiciones o las propiedades de este, deben ser iguales en todas las direcciones del lugar.

La Teoría de Christaller sienta las bases que explica "la organización de las redes urbanas", es decir la organización de una ciudad, gracias a esta teoría podemos establecer las pautas de ordenación de los núcleos urbanos, donde aparecen los llamados "centros de servicios" que brindan mercaderías (bienes y servicios) a la población residente o que circundan por el lugar, este intercambio permite la generación de mercados, que generan rentas, y donde este flujo circular de rentas se distribuyen y enriquecen a la población relacionada directa o indirectamente con esos centro de servicios. Es importante la inversión en la mejora y acondicionamiento de la ciudad para generar focos para la atracción de inversiones nacionales y extranjeras.

\section{SOBRE EL ORDEN DE LAS CIUDADES Y LA JERARQUIZACIÓN DEL ESPACIO.}

¿Pero porque se llama la teoría de los lugares centrales? Esto se debe a que asume que allí donde se prestan servicios se acercan las personas para obtenerlo. De esta manera explica la aparición de un punto en el espacio que organiza el territorio en torno a sín ${ }^{38}$. Aquí me gustaría agregar que "Los patrones de comportamiento de consumo de los agentes que viven o circulan por ahí son lo que determinan la aparición de estos CENTROS DE SERVICIOS que brindan "satisfactores" y el comercio genera e incrementa las posibilidades de crecimiento económico del lugar".

38 Debemos considerar que la localización de los espacios con características semejantes en una región y la distribución denota los atributos esenciales donde se alude el orden del espacio que permite comprender sus cualidades regulares pero por otra parte, también a las posibilidades del desorden del espacio propio del movimiento y transformación del mismo.

39 Se entiende por satisfactor a todo bien de consumo que cubre necesidades para el ser humano. Los satisfactores son las cosas materiales que nos facilitan la vida. En economía y sociología se habla de satisfactor siempre que exista una necesidad a cumplir, estas necesidades pueden ir desde las más básicas hasta las más sofisticadas del hombre contemporáneo. 
La teoría de los lugares centrales explica tres interrogantes; la primera es ¿existen leyes que determinan el número, tamaño y distribución de la población?, la segunda pregunta es ¿por qué se generan poblaciones grandes y pequeñas?, y finalmente ¿Por qué están distribuidas tan irregularmente?

Imagen $\mathbf{N}^{\circ} \mathbf{1}$ : Supongamos en esta imagen que la población se reparte en este lugar e igualmente se distribuye para vivir, podemos suponer que la población que se encuentra en este bloque tiene similitudes en cuanto a los ingresos, gustos y preferencias, todos ellos compiten generando orden o desorden. Podríamos decir que las condiciones de infraestructura en la que viven, pueden ser similares. Concluyo diciendo que si el espacio es isotrópico todos se encuentran en las mismas condiciones para satisfacer sus necesidades.

Área

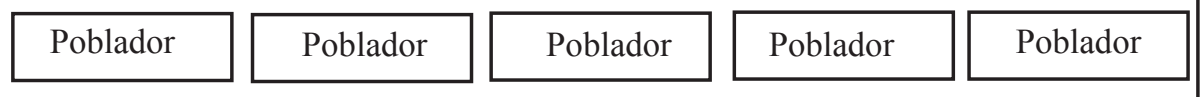

Fuente propia.

Respecto de la primera pregunta que hicimos debemos responder que la población se reúne en base a sus necesidades; debido a esto, aparecerán en este punto de reunión especuladores que ofrecerán los "satisfactores" siempre que la población demandante pueda pagar el precio. Por otro lado el número, el tamaño y la distribución estarán acondicionados por el ofertante de los "satisfactores". La respuesta a la segunda pregunta sobre el origen de una gran población; se debe a las condiciones que existen en el lugar; aquí es importante destacar los recursos ubicuos ${ }^{40}$ y los recursos localizados ${ }^{41}$ y por otro lado las características que posean los pobladores para poder situarse y establecerse en ese lugar o en todo caso debido a la necesidad de satisfacer su necesidad por lo cual deciden establecerse en ese lugar, esto explica que a pesar de no vivir ahí, forman parte de una población flotante que incurre al lugar para poder satisfacer sus necesidades.

La respuesta a la tercera pregunta sobre la distribución irregular se explica dado que si existe un agente ofertante quien tiene la necesidad de maximizar su ganancia, determinara la distribución del espacio a vender, esto implica planificación para crear las condiciones del orden para la distribución. Pero si este no existe; este dependerá de la competencia que existe entre los demandantes lo cual puede fomentar el desorden en la distribución, lo que no significa, que se fomente el orden entre ellos y se pueda dar paso a la distribución por consenso.

40 Son aquellos que pueden localizarse en cualquier parte pero son muy necesarios como el agua y la arena.

41 Son aquellos que solo se encuentran en un determinado punto pero son esenciales para un proceso productivo. 


\section{SOBRE LA NECESIDAD DE INVERSIÓN Y MEJORA DE LA INFRAES- TRUCTURA EN LA CIUDAD.}

Volviendo a las primeras condiciones de la teoría de los lugares centrales nos hablan de un espacio Isótropo y para lograrlo debo establecer que el lugar a desarrollar debe reunir iguales condiciones de infraestructura en toda su longitud, hoy en día si es posible construir, remodelar o adecuar la infraestructura a las necesidades de la población residente y la circundante, así como también replicar cualquier condición ambiental, claro esto dentro un lugar que se acondicione y se controle.

En Alemania existe un hotel a 60 kilómetros de Berlín donde a pesar del clima invernal los huéspedes pueden sumergirse en las cálidas arenas de playa artificial donde un grupo de inversores de Malasia que viendo las dimensiones y ubicación de una enorme estructura de un gigantesco hangar procedente de los tumultuosos tiempos de la guerra fría, y que hasta el día de hoy es uno de los más grandes del mundo Las dimensiones del hangar son tan espectaculares y comprenden 360 metros de largo, 210 metros de ancho y hasta 107 metros de altura. Teniendo en cuenta que la altura total de la Estatua de la Libertad, desde la base del suelo hasta la antorcha, es de 93 metros, usted puede hacerse una idea del tamaño de este hangar capaz de albergar en su interior hasta 12 estatuas completas como la célebre imagen de Manhattan. Junto al hangar se ha construido un hotel con 200 habitaciones llamado, como no, Tropical Islands y cuenta con una playa artificial que puede albergar hasta 6.000 bañistas al día. Con una temperatura perfectamente controlada de $26^{\circ} \mathrm{C}$ y una humedad del $40 \%$, los bañistas pueden perder el miedo a pegarse un chapuzón puesto que las enormes piscinas están climatizadas a $28^{\circ} \mathrm{C}$.

Imagen N²: Muestra el clima invernal de Berlín a las afueras del hotel, el acondicionamiento del hangar y su transformación en Hotel Tropical Island.
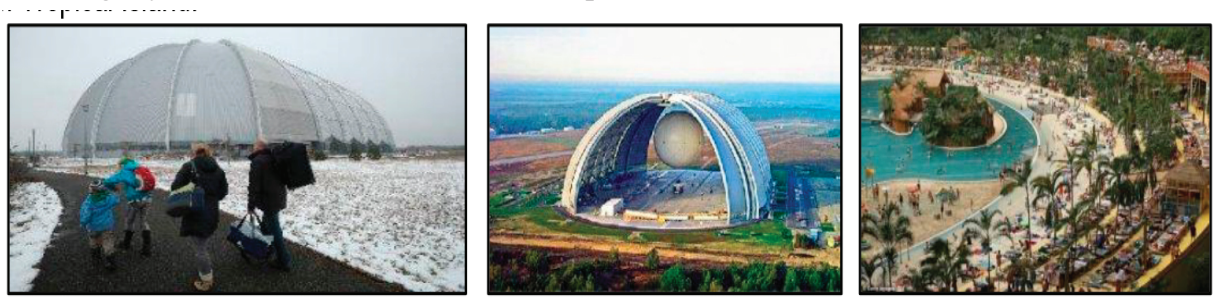

Fuente Yahoo noticias ${ }^{42}$.

Según la teoría de los lugares centrales esta concentración genera fuerzas centrípetas como trabajo, inversión, gasto público y conglomeraciones de proveedores además de cadenas de producción complementarias donde la renta que se genera por este lugar central es aprovechada por la ciudad, la región y el país.

42 https://es-us.noticias.yahoo.com/blogs/blog-de-noticias/construyen-un-para\%C3\%ADso-tropical-en-un-hangar-de-alemania-171019229.html 


\section{LA PLANIFICACIÓN DE LA POBLACIÓN EN UNA CIUDAD}

Debemos hablar sobre la necesidad de planificar para lograr que exista una distribución homogénea de la población, es decir la cantidad personas que reside dentro de un lugar, pero esto es una utopía, debido a que el control podría ocasionar nuevos equilibrios y variaciones de los precios en los diferentes mercados del lugar. Sin embargo la necesidad de control sobre la cantidad de la población es imprescindible debido a la limitada oferta de los recursos ubicuos y que son necesarios para promover y mejorar la calidad de vida de la población residente y circundante.

Con la aparición de lugares centrales y promoviendo la adecuación de la infraestructura del lugar para que responda a todas las necesidades, sin un control debido, permite promover saturación sobre el espacio y genera la aparición fuerzas centrífugas para la conglomeración industrial y urbana. Debido a que se vuelva imposible la atención de las necesidades de la población residente y circundante, genera caos que crean las condiciones para ahuyentar a los consumidores y a los empresarios.

Esto genera una hipótesis en la que puedo afirmar que cuanto mayor es la cantidad de la población residente y circulante; mayor deberá ser las prestaciones para mantener el orden y "status quo" dentro de una determinada locación.

La población residente y circundante en un lugar puede tener similares niveles de ingreso o renta y por tanto coincidir en los gustos y preferencias de los bienes y servicios. Cuando existe orden y se tiene un pleno conocimiento de la renta y las necesidades. Tanto productores como demandantes tienen un perfecto conocimiento del mercado y actúan de manera perfectamente racional.

\section{LA POBLACIÓN DEMANDANTE, EL UMBRAL DE DEMANDA NECESARIO PARA LA GENERACIÓN DE MERCADOS.}

Toda empresa que ofrezca un servicio necesita una cantidad de población mínima que solicite su producto para poder tener los ingresos que le mantienen como negocio. A esto le denominamos el UMBRAL DE DEMANDA. Esto genera el siguiente axioma "cuanto más caro o especializado sea el servicio mayor es la población mínima que necesita tener alrededor para asegurarse ese umbral". Aquí concluimos que para un determinado espacio que contiene a una población, el numero de lugares centrales que ofrecen servicios caros o especializados es menor que aquellos que ofrecen servicios baratos. De esta manera el numero de hospitales, asesorías jurídicas será menor que kioscos, tienda de abarrotes, bares y panaderías.

Otra variable importante dentro de esta teoría es el llamado ALCANCE FÍSICO o rango ideal del mercado. Esta es la distancia máxima que el consumidor está dispuesto trasladarse para consumir un bien o servicio específico 
Imagen $\mathbf{N}^{\circ}$ 3: En la siguiente imagen se muestra el umbral de la demanda y el alcance físico dimensiones necesarias para la demanda total para una determinada empresa.

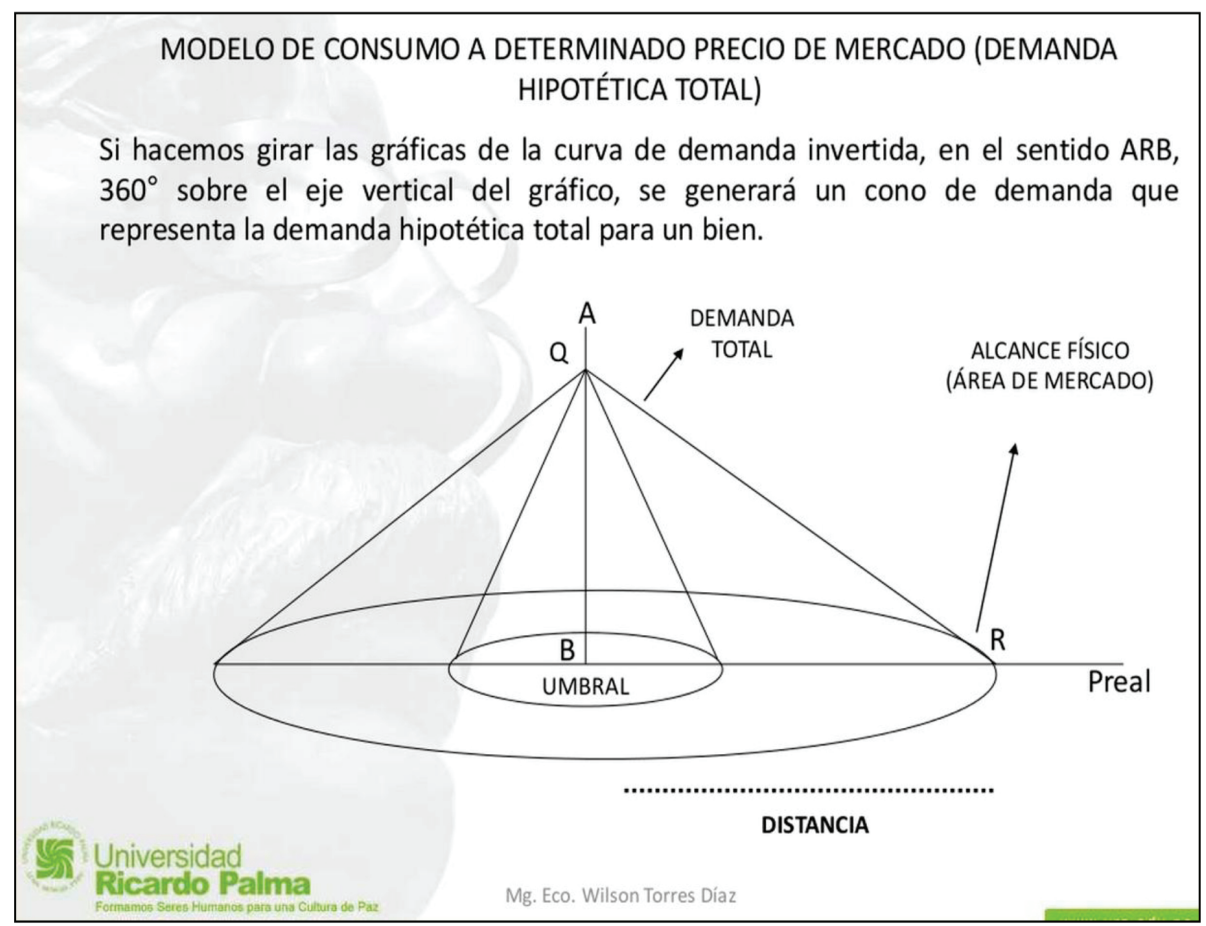

Fuente propia.

De la misma manera que antes, un consumidor está dispuesto a desplazarse más lejos para obtener servicios más caros o especializados que para los servicios normales. Un ejemplo de ello es que un consumidor está dispuesto a desplazarse muchos kilómetros y durante muchas horas para ir a un hospital, pero no para comprar una pieza de pan. Concluyo diciendo que si la infraestructura es la adecuada podemos permitir la movilidad de población entre los diferentes mercados permitiendo la competitividad.

\section{LA FÓRMULA QUE ESTABLECE LA EXISTENCIA DEL LUGAR CENTRAL}

Si la Producción localizada está en función del consumo localizado, de la inversión localizada y del gasto localizado que genera exportación de productos y servicios así como la importación de productos que no se generan en el mercado domestico localizado.

$$
Y_{L}=C_{L}+I_{L}+G_{L}+X_{L}-M_{L}
$$

Si la función de exportación es el índice de Exportaciones Netas y esta es igual a la cantidad de Exportaciones - Propensión a exportar y la función de Importación 
es igual a la propensión a importar + propensión a importar en su relación con la producción más la propensión a exportar producto de las Exportaciones. Al introducir la propensión marginal de la exportación a importar nos da:

$$
X_{L}-M_{L}
$$

$\left(X-m_{x} X\right)-\left(m_{0}+m Y+m_{x} X\right)$ donde la $X$ es producto de la propensión a exportar

Si esto es cierto; la producción localizada dependerá del Consumo autónomo localizado que impulsa a la propensión a consumir localizada y dependiente del nivel de salario más la Inversión autónoma localizada impulsado por la propensión a invertir localizada que será reactiva inversamente a las tasas de interés pero atraída positivamente por la movilización de Capital Invertido más el Gasto autónomo realizado en construcción y mejora de la infraestructura necesaria en el lugar central que es dependiente del nivel de impuestos y renta menos la propensión a importar más la propensión a importar que es generada por la producción más la propensión a exportar menos regalías y utilidades (mf) ya que en muchos sectores exportadores predomina el capital foráneo que representan fugas.

$Y_{L}=\left(c_{o}+\beta c_{1}, w^{+}\right)+\left(I_{0}+\beta I_{1}, i^{-}, K^{+}\right)+\left(G_{0}+\beta G_{1}, t^{+}, y^{+}\right)-\left(m_{0}+m Y-m_{x} X\right)-m f\left(X-m_{x} X\right)$

Una vez más se infiere que el tamaño de $m_{x}$ reflejaría el grado de desarrollo regional, y se supone que la relación entre éste con el valor de $m_{x}$ es inversa. Lo que de nuevo muestra el rezago imperante en la base doméstica. La formación de un lugar central estará en base a la satisfacción de necesidades de la población que es residente y de la que es circundante.

\section{LA INFLUENCIA DE LOS COSTES DE PRODUCCIÓN Y DE DISTRIBUCIÓN EN LA FORMACIÓN DE LOS LUGARES CENTRALES}

Muchas de las Inversiones son producto de los costos fijos y variables que asumen los emprendedores que podrían ser entendidos como los costes de producción y de los costes de distribución

$$
\mathrm{Y} \Rightarrow \mathrm{I}=\mathrm{Q} \Rightarrow \mathrm{f}(\mathrm{CP}+\mathrm{CD})
$$

Si los Costos Variables (CV) influyen en los Costes de Producción (CP) y de los Costes de Distribución (CD), Si los costes de Distribución (CD) están en función de la distancia (d) y del precio de transportarse (F). Y el precio de trasportarse o precio generalizado asume costes, tiempo y condiciones tales como la comodidad y seguridad. 


$$
\begin{gathered}
\mathrm{CP}=\mathrm{CF}+C V \\
\mathrm{CD}=\mathrm{f}\{\mathrm{d}, \mathrm{F}\} \\
\mathrm{F}=\mathrm{p}+\mathrm{vt}+\theta
\end{gathered}
$$

Donde: $\mathrm{p}=$ Viaje

$\mathrm{v}=$ Coste del tiempo

$\mathrm{t}=$ tiempo

$\theta=$ comodidad y seguridad

Por tanto, la producción depende de los costos de producción y de distribución; y esta a su vez depende del viaje, del coste de tiempo y del tiempo más la comodidad y seguridad que asumen los que trasladan los factores de producción.

\section{LAS NECESIDADES Y SU CLASIFICACIÓN DE ALTO ORDEN Y DE BAJO ORDEN}

La creación de los lugares centrales se crean en base a las necesidades de la población por lo que es importante conocerlas, Christaller afirma que si estas mercancías y servicios son de acuerdo a necesidades de alto orden y de bajo orden. Son necesidades de alto orden aquellas que requieren de grandes umbrales y grandes áreas de mercado y son necesidades de bajo orden aquellas que requieren de umbrales y áreas de mercado pequeñas.

Dado que pueden existir mercancías y servicios de alto orden, podemos suponer ahora un sistema de dos funciones, una de bajo orden (X) y otra de alto orden (Y), y suponemos que $\mathrm{Y}$ requiere de un área de mercado 3 veces mayor que $\mathrm{X}$, y que esto satisface el umbral de mercado para la función Y.

Las necesidades crea tres categorías: el primero de ellos es el de lugar central, el cual es un núcleo de concentración de población que ofrece bienes y servicios con un nivel de especialización a un área mayor de influencia que la ocupada físicamente por dicho núcleo

El segundo concepto es bien o servicio central que consiste en los bienes y servicios con un nivel de especialización que son ofrecidos solamente en los lugares centrales; y

Finalmente, región complementaria también denominada área de influencia o hinterland que es el área que abastece un lugar central de bienes y servicios centrales.

Sobre estas necesidades debo decir que debemos ser capaces de identificarlos para poder ofrecerlas y satisfacerlas. Una empresa en un lugar tiende a concentrar la población y a servir a los habitantes del lugar central y a todos sus clientes que tengan un alcance físico del mercado. Existen áreas no cubiertas por la empresa 
y la población más alejada tiene la tendencia a no desplazarse para adquirir un servicio. Para estos muchas empresas modifican su distribución o su área de alcance haciéndola hexagonal que garantice la prestación de este servicio. Sin embargo es función del estado el que debe procurarlas.

Imagen $\mathbf{N}^{\circ}$ : Esta imagen explica la organización jerárquica de los espacios alrededor del lugar central todos ellos tratando de satisfacer las necesidades de la población residente y circundante.

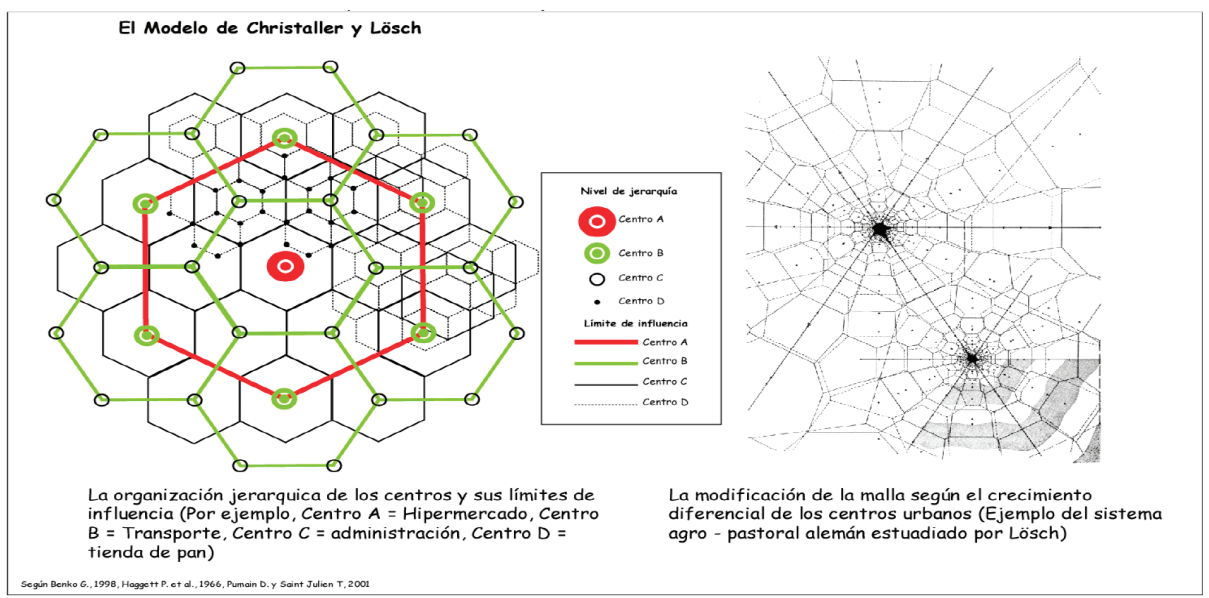

Fuente Central Places in Southern Germany de Walter Christaller.

Si esto es verdad debemos adaptar el entorno para que un mayor número de empresas encuentren interesante invertir en un determinado lugar más allá de los nichos de mercado.

\section{CONCLUSIONES}

1. Se pueden generar lugares centrales donde la inversión en infraestructura cree las condiciones en la ciudad para su crecimiento.

2. Las condiciones de infraestructura deben ser iguales en cada punto del plano que permitan las condiciones de acontecimiento, cambio y movilidad.

3. La información debe llegar a tiempo para que la población pueda actuar de acuerdo a las condiciones de cambio que se generan.

4. Los servicios de infraestructura deben ser proporcionales al tamaño de la población.

5. Los costos de la infraestructura deben ser soportados sobre la base tributaria.

6. Si estamos interesados en la formación de lugares centralizados para el desarrollo de las ciudades y de la región esta dependerá de la producción localizada fomentada por el consumo autónomo y la propensión a consumir debido a la relación con los niveles de renta que se impulsa 
debido a los niveles de inversión autónoma que existe y la propensión a invertir debido a condiciones como bajo niveles de tasa de interés y una mayor disponibilidad de capital que puede ser entendido como gastos en construcción e infraestructura que ha sido impulsado por el gobierno y la intensión autónoma de gastar y su propensión a gastar debido a la relación directa con una mayor recaudación de impuestos y el mayor nivel de producción disminuido por los niveles de importación autónoma que no satisface el mercado domestico y el aumento de la propensión a importar de sus habitantes pero que mejora debido a la propensión a exportar de sus habitantes producto del grado de desarrollo localizado y regional sin embargo como no todas las inversiones son producto del capital invertido por extranjeros se percibe como fuga de capitales producto de las pagos al capital extranjero invertido.

\section{REFERENCIAS BIBLIOGRÁFICAS / WEBGRAFÍA}

1. Pike Andy, Rodríguez-Pose Andrés, Tomaney John (2011). Desarrollo local y regional. Traducido por Joan Noguera Tur. Universitat de València, 400 páginas.

2. García-Bermejo Ochoa Juan C. (2009). Sobre la economía y sus métodos. Editorial CSIC - CSIC Press. 552 páginas.

3. Hernández Aragón, Julia. (2009). La localización de las Actividades de Los Servicios Superiores en el Centro de la Ciudad: Un Análisis Estático del Patrón de Localización de los Bancos y Servicios Especializados en la Ciudad de Puebla.

4. Walter Christaller. (1966) Central Places in Southern Germany. Traducido por Carlisle W. Baskin Prentice-Hall. 230 páginas.

5. http://espacio-geografico.over-blog.es/article-distribucion-108513576. html

6. http://es.slideshare.net/septimio/3-localizacion-distribucionrelacion-einteraccio

7. http://www.eumed.net/libros-gratis/2007a/249/1.htm

8. https://es-us.noticias.yahoo.com/blogs/blog-de-noticias/construyenun-para\%C3\%ADso-tropical-en-un-hangar-de-alemania-171019229. html 